\title{
The Research of Some Single-Machine Scheduling Problems
}

\author{
Zhigao Liao \\ Department of Management \\ Guangxi University of Science and Technology \\ LiuZhou, China
}

\author{
Xiaojing Zhao \\ Department of Management \\ Guangxi University of Science and Technology \\ LiuZhou , China
}

\begin{abstract}
In this paper, we develop a new scheduling model with learning effects where the actual processing time of a job is not only a function of the total normal processing times of the jobs already processed, but also a function of the job's scheduled position. We show that the single-machine scheduling problems to minimize make span and total complete time are still polynomially solvable under the proposed model. We further show that the single-machine scheduling problems minimizing the weighted sum of completion times and minimizing the maximum lateness are also polynomially solvable under certain conditions.
\end{abstract}

Keywords-Scheduling; position-dependent learning effect model; time-dependent learning effect mode

\section{INTRODUCTION}

Scheduling problems have received considerable attention since the middle of the last century. However, most researchers assume that job processing times are known and fixed during the whole processing process. Recent empirical studies in several industries have demonstrated that unit costs decline as companies produce more of a product and gain knowledge or experience. This phenomenon is well-known as the "learning effect" in the literature (see,e.g., [1,2]).

Biskup (see,e.g., [2]) was the first to investigate the learning effect in scheduling problems. He assumed that the time needed to perform an operation decreases by the number of repetitions., the processing time of a job is a function of the job position in a sequence. In the classical position-dependent learning effect model, the actual processing time $p_{i r}$ of job $J_{i}$ when it is scheduled in the $\mathrm{rth}$ position in a processing sequence is defined as

$$
p_{i r}=p_{i} r^{a}
$$

where $\mathrm{a}$ is a non-positive learning index and $p_{i}$ denotes the normal processing time of job $J_{i}$. Under this assumption, the learning effect of a job only depends on the number of jobs that are scheduled in front of it. It implies the learning rates of all jobs are all the same. There are several studies in this context. Biskup showed that single-machine scheduling problems with a learning effect still remain polynomially solvable if the objective is to minimize the deviation from a common due date or to minimize the sum of flow times.

Kuo and Yang (see,e.g., [3]) considered a single-machine scheduling problem with a time-dependent learning effect. There are $n$ jobs to be processed on a single machine. Each of them is available at time zero. Let $p_{i}$ be the normal (sequence-independent) processing time of job $J_{i}$ in a sequence and $p_{[k]}$ be the normal processing time of a job if scheduled in the kth position in a sequence. The normal processing time of a job is incurred if the job is scheduled first in a sequence. The processing times of the following jobs are smaller than their normal processing times because of the learning effect. To introduce the notion that the more you practice, the better you learn, we define a time-dependent learning effect as follows. Let $p_{i r}$ be the actual processing time of $J_{i}$ if it is scheduled in position $r$ in a sequence. Then

$$
p_{i r}=p_{i}\left(1+\sum_{k=1}^{r-1} p_{[k]}\right)^{a}
$$

where $a(a \leq 0)$ is a constant learning index. Kuo and Yang proved that the total completion time and minimization problem is still polynomially solvable under the single-machine.

Based on the two models of Biscup, Kuo and Yang, in this paper, we develop a new scheduling model with learning effects where the actual processing time of a job is not only a function of the total normal processing times of the jobs already processed, but also a function of the job's scheduled position. Based on this new learning model, we need search that the single-machine scheduling problems to minimize makespan and total complete time are still polynomially solvable under the proposed model. We also need search that the single-machine scheduling problems minimizing the weighted sum of completion times and minimizing the maximum lateness are also polynomially solvable under certain conditions.

\section{SOME SINGLE MACHINE PROBLEMS}

There are given a single machine and $\mathrm{n}$ independent and non-preemptive jobs that are immediately avail-able for processing. The machine can handle one job at a time and preemption is not allowed. Let $p_{i}$ be the normal processing time of job $J_{i}$ and $P_{[k]}$ be the normal processing time of a job if it is scheduled in the kth position in a sequence. Associated with each job $J_{i}(\mathrm{i}=1,2, \ldots, \mathrm{n})$ is a weight $w_{[k]}$, a due date $d_{[k]}$ and 
a completion date $C_{[k]}$. Let $p_{j r}$ be the actual processing time of $J_{j}$ if it is scheduled in the rth position in a sequence, then

$$
p_{j r}=p_{j}\left(\alpha a^{\sum_{k=1}^{r-1} \ln p_{[k]}}+\beta\right) r^{b}
$$

where $\alpha \geq 0, \beta \geq 0,0 \leq a \leq 1, b<0$ are some indexes which we can make it ourslves. For the sake of convenience, we make the above learning model as $L E$. Obviously, in the model (4), the actual processing time of a job depends not only on a function of the total normal processing times of the jobs already processed, but also a function of the job's scheduled position. The learning effect will be more obvious if a job's scheduled position is later.For a given feasible scheduling, $C_{\max }=\max _{1 \leq i \leq n}\left\{C_{j}\right\}$ and $\sum C_{j}$ denote the makespan and total completion time, $\sum w_{j} C_{j}$ and $L_{\max }=\max _{1 \leq j \leq n}\left\{L_{j}\right\}$ denote total weighted completion time and the maximum delay. Besides, $L_{j}(S)=C_{j}(S)-d_{j}$. In the sequel, we will adopt the three-field notation scheme $\alpha|\beta| \gamma$.

\section{SOME SINGLE MACHINE PROBLEMS}

First, we give a lemma.it is useful for the following theorems.

\section{Lemma}

1.

$$
\begin{aligned}
& (u-1)(x+\beta)+v\left(x a^{y}(\ln u-u)\right. \\
& +\beta(1-u)) \geq 0 \\
& u \geq 0<v \leq 1 x \geq 0 \\
& y \geq 10<\alpha \leq 1 \beta>0 \\
& F(u)=(u-1)(x+\beta)+
\end{aligned}
$$

If

Proof. If

$$
v\left(x a^{y}(\ln u-u)+\beta(1-u)\right)
$$

Then the first derivative about $u$ is

$$
\begin{aligned}
& F^{\prime}(u)=x+\beta+v\left(x a^{y}\left(\frac{1}{u}-1\right)-\beta\right) \\
& =x\left(1-\left(1-\frac{1}{u}\right) v a^{y}\right)+\beta(1-v) \geq 0
\end{aligned}
$$
where $0 \leq\left(1-\frac{1}{u}\right) v a^{y} \leq 1$.We have $F(u)$
progressive increases about $\mathrm{u}$, therefore $F(u) \geq F(1)=0$.

First, we study the single machine makespan minimization problem under model(3), denoted by 1| $L E \mid C_{\max }$. And we show that an optimal schedule for the problem can be obtained by the Shortest Processing Time first (SPT) rule. The result is stated as follows.
Theorem 1. For the problem $1|L E| C_{\max }$, there exists an optimal schedule in which the jobs are ordered according to the SPT rule.

Proof. Suppose that there exists an optimal schedule $S^{\prime}=\left(\pi, J_{j}, J_{i}, \pi^{\prime}\right)$ with $P_{i}<P_{j}$,

where $\pi$ and $\pi^{\prime}$ denote the partial sequences of $S^{\prime}$. Let $S$ be a schedule with jobs $J_{j}$ and $J_{i}$ of $S$ mutually exchanged, that is, $S=\left(\pi, J_{i}, J_{j}, \pi^{\prime}\right)$. In addition, we assume that there are $\mathrm{r}-1$ jobs in $\pi$. Let $C_{j}(S)$ and $C_{j}\left(S^{\prime}\right)$ respectively denote the complete times of job $J_{j}$ in $S$ and $S^{\prime}$ and let $C_{i}(S)$ and $C_{i}\left(S^{\prime}\right)$. We will show that the interchange of jobs $J_{j}$ and $J_{i}$ does not influence the objective value. The repeated implementation of this argument will lead to the optimality of the SPT rule for the problem $1|L E| C_{\max }$. To further simplify the notation, let $t$ denote the completion time of the last job $\pi$. Besides, the completion time of the last job $\pi^{\prime}$ does not influence the two schedules. Then from the Eq. (3) the completion of job $J_{i}$ and $J_{j}$ in $\mathrm{S}$ are

$$
C_{i}(S)=t+p_{i}\left(\alpha a^{\sum_{k=1}^{r-1} \ln p_{[k]}}+\beta\right) r^{b}
$$

and

$$
\begin{aligned}
& C_{j}(S)=t+p_{i}\left(\alpha a^{\sum_{k=1}^{r-1} \ln p_{[k]}}+\beta\right) r^{b}+ \\
& p_{j}\left(\alpha a^{\sum_{k=1}^{r-1} \ln p_{[k]}+\ln p_{i}}+\beta\right)(r+1)^{b}
\end{aligned}
$$

also in $S^{\prime}$ are

$$
C_{j}\left(S^{\prime}\right)=t+p_{j}\left(\alpha a^{\sum_{k=1}^{r-1} \ln p_{[k]}}+\beta\right) r^{b}
$$

and

$$
\begin{aligned}
& C_{i}\left(S^{\prime}\right)=t+p_{j}\left(\alpha a^{\sum_{k=1}^{r-1} \ln p_{[k]}}+\beta\right) r^{b}+ \\
& p_{i}\left(\alpha a^{\sum_{k=1}^{r-1} \ln p_{[k]}+\ln p_{j}}+\beta\right)(r+1)^{b}
\end{aligned}
$$

Thus we have

$$
C_{i}\left(S^{\prime}\right)-C_{j}(S \neq
$$


$p_{i} r^{b}\left(\left(\frac{p_{j}}{p_{i}}-1\right)\left(\alpha a^{\sum_{k=1}^{r-1} \ln p_{[k]}}+\beta\right)\right.$

$+\frac{(r+1)^{b}}{r^{b}}\left[\alpha a^{\sum_{k=1}^{r-1} \ln p_{[k]}}\left(a^{\ln p_{j}}-\frac{p_{j}}{p_{i}} a^{\ln P_{i}}\right)\right.$

$\left.\left.+\beta\left(1-\frac{p_{j}}{p_{i}}\right)\right]\right)$

Let

$u=\frac{p_{j}}{p_{i}}, v=\frac{(r+1)^{b}}{r^{b}}, x=\alpha a^{\sum_{k=1}^{r-1} \ln p_{[k]}}, y=\ln p_{i}$ clearly $u \geq 1,0 \leq \frac{(r+1)^{b}}{r^{b}} \leq 1, x \geq 0, y \geq 1$.

Thus, by Lemma 1 we have

$$
\begin{aligned}
& C_{i}\left(S^{\prime}\right)-C_{j}(S)= \\
& p_{i} r^{b}((u-1)(x+\beta) \\
& \left.+v\left[x\left(a^{\ln u+y}-u a^{y}\right)+\beta(1-u)\right]\right) \geq 0
\end{aligned}
$$

Consequently, $C_{j}(S) \leq C_{i}\left(S^{\prime}\right)$. this completes the proof.

Now we sthudy the single machine total complete time problem under model(3), denoted by $1|L E| \sum C_{j}$. And we show that an optimal schedule for the problem can be obtained by the Shortest Processing Time first (SPT) rule. The result is stated as follows.

Theorem 1. For the problem $1|L E| \sum C_{j}$, there exists an optimal schedule in which the jobs are ordered according to the SPT rule.

Proof. The marks in theorem 1 are used in here. According to the proof of theorem 1, we just need to prove $\mathrm{C}_{i}(S)+\mathrm{C}_{j}(S) \leq \mathrm{C}_{j}\left(S^{\prime}\right)+\mathrm{C}_{i}\left(S^{\prime}\right)$. Because of $P_{i}<P_{j}$, the equation

$$
C_{i}(S)-C_{j}\left(S^{\prime}\right)=
$$

$$
r^{b}\left(\alpha a^{\sum_{k=1}^{r-1} \ln P_{[k]}}+\beta\right)\left(P_{i}-P_{j}\right) \leq 0
$$

is right clearly, that is, $C_{i}(S) \leq C_{j}\left(S^{\prime}\right)$.besides, the proof of $C_{j}(S) \leq C_{i}\left(S^{\prime}\right)$ is ready in theorem 1.then we have $C_{i}(S)+C_{j}(S) \leq C_{j}\left(S^{\prime}\right)+C_{i}\left(S^{\prime}\right)$.

This completes the proof.

The single-machine scheduling problems minimizing the weighted sum of completion times, that is, $1 \| \sum w_{j} C_{j}$ is a very important part. In the classical scheduling, an optimal schedule for the problem can be obtained by the Weighted Shortest Processing Time first (WSPT) rule. But the conclusion is not always right under model (3). However, under certain conditions the problems can be solved in polynomial time under model (3).

Theorem 3. For the problem $1 \| \sum w_{j} C_{j}$, if jobs have reversely agreeable weights, that is, $p_{i} \leq p_{j} \Rightarrow w_{i} \geq w_{j}$ for all jobs $J_{i}$ and $J_{j}$, then there exists an optimal schedule in which the jobs are ordered in non-decreasing order of $\frac{w_{j}}{p_{j}}$ (WSPT rule).

Proof. The marks in theorem 1 are also used in here. Supposing that here is an optimal schedule $S^{\prime}=\left(\pi, J_{j}, J_{i}, \pi^{\prime}\right)$ which follow the WSPT rule, that is, there exist job $J_{i}$ and job $J_{j}$ with $\frac{w_{j}}{p_{j}} \leq \frac{w_{i}}{p_{i}}$. Note that the weights and the normal processing time of the all jobs and are reverse. Thus, we have $p_{i} \leq p_{j}$ and $w_{i} \geq w_{j}$. We get $C_{i}(S) \leq C_{j}\left(S^{\prime}\right)$ and $C_{j}(S) \leq C_{i}\left(S^{\prime}\right)$ from theorem 1 and theorem 2 respectively. We can have

$$
\begin{aligned}
& w_{j} C_{j}\left(S^{\prime}\right)+w_{i} C_{i}\left(S^{\prime}\right)-w_{i} C_{i}(S)-w_{j} C_{j}(S) \\
& \geq w_{i} C_{j}(S)+w_{j} C_{i}(S)-w_{i} C_{i}(S)-w_{j} C_{j}(S) \text { th } \\
& =\left(C_{i}(S)-C_{j}(S)\right)\left(w_{i}-w_{j}\right) \geq 0,
\end{aligned}
$$

at is, $w_{i} C_{i}(S)+w_{j} C_{j}(S) \leq w_{j} C_{j}\left(S^{\prime}\right)+w_{i} C_{i}\left(S^{\prime}\right) \quad$ This completes the proof.

Next, we will show that the single machine problem to minimizing the maximum lateness under model(3), denoted by $1|L E| \sum L_{\max }$, can be solved if the job processing times and the due dates are agreeable, that is, $d_{i} \leq d_{j}$ implies $p_{i} \leq p_{j}$ for all jobs $J_{i}$ and $J_{j}$.

Theorem 4. If the normal processing times and the due dates of job are agreeable, namely, that $d_{i} \leq d_{j}$ implies $p_{i} \leq p_{j}$ is true for jobs $J_{i}$ and $J_{j}$, then the Earliest Due Date first rule (EDD rule) is optimal for the problem $1|L E| \sum L_{\max }$.

Proof. The marks in theorem 1 are still used in here. We suppose that job $J_{i}$ and $J_{j}$ satisfy $d_{j} \leq d_{i}$ in optimal scheduling $S^{\prime}=\left(\pi, J_{j}, J_{i}, \pi^{\prime}\right)$. The normal processing times and the due dates of job are agreeable, then we have $p_{i} \leq p_{j}$. Besides, we get $C_{i}(S) \leq C_{j}\left(S^{\prime}\right) \quad$ and $\quad C_{j}(S) \leq C_{i}\left(S^{\prime}\right) \quad$ from theorem 1 and theorem 2 respectively. By definition, the lateness of jobs $J_{i}$ and $J_{j}$ in $\mathrm{S}$ and jobs $J_{i}$ and $J_{j}$ in $S^{\prime}$ are respectively

$$
\begin{aligned}
& L_{j}(S)=C_{j}(S)-d_{j} \\
& L_{i}(S)=C_{i}(S)-d_{i} \\
& L_{i}\left(S^{\prime}\right)=C_{i}\left(S^{\prime}\right)-d_{i} \\
& L_{j}\left(S^{\prime}\right)=C_{j}\left(S^{\prime}\right)-d_{j} \\
& L_{j}(S) \leq L_{i}\left(S^{\prime}\right) \text { can be obtained from } C_{j}(S) \leq C_{i}\left(S^{\prime}\right)
\end{aligned}
$$
and $d_{i} \leq d_{j}$.Also, since $C_{i}(S) \leq C_{i}\left(S^{\prime}\right)$,we have 
$L_{i}(S) \leq L_{i}\left(S^{\prime}\right)$

.Then $\max \left\{L_{i}(S), L_{j}(S)\right\} \leq \max \left\{L_{j}\left(S^{\prime}\right), L_{i}\left(S^{\prime}\right)\right\}$ This completes the proof.

\section{CONCLUSIONS}

In this paper, we develop a new scheduling model with learning effects where the actual processing time of a job is not only a function of the total normal processing times of the jobs already processed, but also a function of the job's scheduled position. We show that the single-machine scheduling problems to minimize makespan and total complete time are still polynomially solvable under the proposed model. We further show that the single-machine scheduling problems minimizing the weighted sum of completion times and minimizing the maximum lateness are also polynomially solvable under certain conditions. These results are sig-nificant extensions of some of the existing results on learning effects in the literature. We believe that the general learning effect model offered here will turn out to be more useful in the theory and applications of scheduling.

\section{REFERENCES}

[1] A.B. Badiru, Computational survey of univariate and multivariate learning curve models, IEEE Transactions on Engineering Management 39 (1992) 176-188

[2] D. Biskup, Single-machine scheduling with learning considerations, European Journal of Operational Research 115 (1999) 173-178.

[3] W.-H. Kuo, D.-L. Yang, Minimizing the total completion time in a single machine scheduling problem with a time-dependent learning effect, European Journal of Operational Research 174 (2006) 1184-1190. 